\title{
Order effects in the rubber hand illusion.
}

\author{
Lush, P.*1,2
}

1 Sackler Centre for Consciousness Science, University of Sussex, Falmer, BN1 9RH, UK

2 Department of Informatics, Chichester Building, University of Sussex, Falmer, BN1 9RH, UK

Data and analysis available at: https://osf.io/d4fmc/

This manuscript contains exploratory analyses of an existing dataset. No analyses were preregistered.

Correspondence: p.lush@sussex.ac.uk 


\begin{abstract}
Seeing a fake hand brushed in synchrony with brushstrokes to a participant's hand (the rubber hand illusion; RHI) prompts reports of referred touch, illusory ownership and that the real hand has drifted toward the fake hand (proprioceptive drift). According to one theory, RHI effects are attributable to multisensory integration mechanisms, but they may alternatively (or additionally) reflect the generation of experience to meet expectancies arising from demand characteristics (phenomenological control). Multisensory integration accounts are supported by contrasting synchronous and asynchronous brush stroking conditions, typically presented in counter-balanced order. This contrast is known to be confounded by demand characteristics, but to date there has been no exploration of the role of demand characteristics relating to condition-order. In an exploratory study, existing data from a rubber hand study (n $=124)$ were analysed to test order effects. Synchronous condition illusion report and the difference between synchronous and asynchronous conditions in both report and proprioceptive drift were greater when the asynchronous condition was performed first (and therefore participants were exposed to the questionnaire materials). These order effects have implications for interpretation of reports of ownership experience: in particular, there was no mean ownership agreement in the synchronous-first group. These data support the theory that reports of ownership of a rubber hand are at least partially attributable to phenomenological control in response to demand characteristics.
\end{abstract}




\section{Order effects in the rubber hand illusion.}

Demand characteristics are cues which inform participants' interpretations of an experimental situation (Orne, 1962; for recent overviews see Corneille \& Lush, 2021; Klein et al., 2012; Sharpe \& Whelton, 2016). Such cues include (but are not limited to) prior beliefs, experimental instructions and recruitment materials and aspects of the experimental procedure itself. According to a recent model of demand characteristics (Corneille \& Lush, 2021) hypothesis-awareness (accurate knowledge of experimental aims; participants may also be hypothesis-mistaken, or hypothesis-unaware) can lead to a range of responses including faking, imagination, reactance and phenomenological control (response to imaginative suggestion according to a stable trait ability; Dienes et al., 2020; Lush et al., 2020). In repeated-measures experiments, cues in the first condition undertaken may increase hypothesis awareness, which can in turn influence responses in the second condition, resulting in systematic order effects. This manuscript presents analyses of order effects in measures of a well-known effect for which repeated-measures designs are often employed the rubber hand illusion (RHI).

Participants report experiences of ownership of (and touch felt on) a fake hand in response to synchronous brushing of a fake hand and their real hand. According to the dominant account, these effects arise from the disruption of multi-sensory integration mechanisms which support attributions of body ownership (Botvinick \& Cohen, 1998; Riemer et al., 2019). Recently it has been proposed that such reports are at least partially attributable to phenomenological control (Lush et al., 2020). On this account, expectancies arising from demand characteristics are met by the control of phenomenology, just as in response to imaginative suggestion (for related accounts of the RHI see Alsmith, 2015; Dieguez, 2018). Claims for a role of multisensory integration in rubber hand effects are primarily supported by contrasting measures between synchronous stroking and an 
asynchronous control condition in which there is a delay between brush strokes on the fake and real hands (see Riemer et al., 2019). Claims for a role of phenomenological control in RHI experience are primarily supported by relationships between response to imaginative suggestion (a stable trait) and rubber hand measures (Lush, Dienes, et al., 2021; Lush et al., 2020). Whether or not there are rubber hand effects beyond those attributable to expectancies remains unknown, because existing control method are flawed (Lush, 2020). Specifically, rubber hand effects are commonly measured by Likert scale agreement ratings for statements describing expected experiences and the difference in estimated hand position before and after stroking ('proprioceptive drift'). For contrasts of synchronous and asynchronous stroking to provide support for multisensory integration theories, all relevant factors must be matched across conditions (see Riemer et al., 2019)). However, participant expectancies are not matched across these conditions; contrasts between them are confounded by hypothesis awareness (Lush, 2020; Lush, Seth, et al., 2021; Reader, 2021).

Typically, the presentation order of synchronous and asynchronous conditions is counterbalanced. Participants are therefore exposed to information relative to hypothesis awareness during the first condition which may inform expectancies for the second condition. For example, a participant might well interpret the statement "I felt as if the rubber hand were my hand" as evidence that the experimenter expects such an experience to occur. Similarly, being asked to provide estimates of the position of their own hidden hand before and after the brush stroking procedure may communicate to participants that a change in perceived hand position is anticipated by the experimenter (see Lush et al., 2021). Systematic differences in demand characteristics between presentation order groups would render counterbalancing ineffective (see Reader, 2021).

In the present study, open data from a the control group of a large-scale rubber hand experiment (Lush et al., 2020) were analysed to test for order effects in agreement scores and 
proprioceptive drift. It is important to note that participants were told that ownership experience is part of the illusion and received descriptions of synchronous and asynchronous procedures. This was so that reports of expectancies for each condition could be reported (participants, however, were not told which condition was expected to generate ownership experience). The knowledge that an experience of ownership is anticipated and reflection on expectancies may have influenced the results of this study. For example, order effects in ownership experience reports may be more pronounced in naive participants, or reflection on expectancies may lead to exaggerated effects. While information about the procedure is not typically provided in RHI studies, neither is it common practice to exclude participants who have prior knowledge of the RHI. Indeed, in some studies participants receive more information than the participants in this sample when they are screened for illusion experience prior to experimental participation (e.g., Chancel et al., 2021). That aside, the results of this study should be considered preliminary, and it will be necessary to establish whether or not effects in this sample differ from effects when participants have no prior knowledge of the RHI.

Because participant expectancies predict rubber hand report (Lush et al., 2020), if participants generate hypothesis awareness (and accompanying expectancies) from demand characteristics in the asynchronous control condition, we should see greater mean response in participants who experience the control (asynchronous) condition first. 


\section{Method}

\section{Participants}

Anonymized open data from 124 participants (99 female, 25 male, mean age $=18.9$, $\mathrm{SD}=1.1$ ) who formed the control group in a previous rubber hand study (Lush et al., 2020) were analysed. The data were split into two groups by condition order. The asynchrony-first group (who performed the control condition before the experimental condition) consisted of 50 participants (Mean age 19.9, $\mathrm{SD}=0.9,47$ female 13 male). The synchrony first group (who performed the control condition after the experimental condition) consisted of 64 participants (Mean age 19.0, $\mathrm{SD}=1.2,52$ female 12 male).

Participants were provided with the following information:

"To perform this illusion, your hand will be hidden from view by a box and a rubber hand will be placed in front of you so that you can see it. The experimenter will use brushes to stroke the rubber hand which you can see, and also your own hand which is out of sight. The combination of information from touch and vision induces an illusory experience of ownership over the rubber hand. You will undergo the illusion procedure in two conditions: when the brush stroke you can see on the rubber hand is synchronous with the brush stroke you can feel on your own hand, and when there is a short delay between the brush stroke on your hand and on the rubber hand"

Participants also answered the following question directly prior to participation:

"How strongly do you expect to feel the rubber hand is your own hand at least a little bit when the brush strokes on your own hand and on the rubber hand are/are not in synchrony?" 


\section{Measures}

See Lush et al., (2020) for details of data collection. Three illusion statements (Botvinick \& Cohen, 1998) were analysed:

S1: It seemed as if I were feeling the touch of the paintbrush in the location where I saw the rubber hand touched.

S2: It seemed as though the touch I felt was caused by the paintbrush touching the rubber hand.

S3: I felt as if the rubber hand were my hand.

S1 and S2 are commonly interpreted as describing experiences of touch felt on the fake hand and S3 is commonly interpreted as describing an experience of ownership over the fake hand (Riemer et al., 2019):

An 'illusion index' score was calculated (mean response to the three statements). Proprioceptive drift was calculated from the difference between pre-and post brush stroking estimates of perceived hand position.

\section{Analyses}

Because this study analyses existing data, no analysis plan was preregistered and the presented analyses should therefore be considered exploratory. To investigate order-effects, ttests of task order differences for both measures between 'asynchrony-first' and 'synchronyfirst' groups were conducted.

To test the impact of order effects on inferences regarding group-level evidence of ownership experience, one-sample t-test of mean scores for each statement were conducted for each group (see supplemental material). 
H1s for Bayes factors were modelled (as preregistered for Lush et al., 2020) based on differences between synchronous and asynchronous conditions across all groups reported by Botan et al., (2018): For agreement statements, approximately 1 unit (on a 7 point scale) and for proprioceptive drift, approximately $1 \mathrm{~cm}$.

95\% CIs (interpreted as Bayesian credibility intervals with a uniform prior) were used to estimate measures. Bayes factors were calculated with the calculator at: https://harrytattan-birch.shinyapps.io/bayes-factor-calculator/. Robustness Regions (RR) were determined as the set of scale factors that led to the same qualitative conclusion (either $B>3$, or $B<1 / 3$; or $1 / 3<B<3$; Dienes, 2019).

Data and analysis available at: https://osf.io/d4fmc/. 


\section{Results}

\section{Proprioceptive drift}

Figure 1 shows mean proprioceptive drift in the two groups. The difference between synchronous and asynchronous condition proprioceptive drift was greater for the asynchronyfirst group $(M=1.2 \mathrm{~cm}, S D=2.5)$ than for the synchrony-first group $(M=0.0 \mathrm{~cm}, S D=2.8)$, $t(121.59)=2.33, p=.022,95 \%$ CI $[.17,2.14], d=.42,95 \% \mathrm{CI}[.06, .77], \mathrm{B}_{\mathrm{H}(0,1)}=7.57$, $\mathrm{RR}_{\mathrm{B}>3}[0.30,4.70]$. There was no sensitive evidence for or against a difference in synchronous condition proprioceptive drift between the asynchrony-first group $(M=1.2, S D$ $=1.4)$ and the synchrony-first group $(M=0.60, S D=2.0), t(121.59)=1.17 p=.24,95 \% \mathrm{CI}$ $[-.38,1.49], d=.2195 \%$ CI $[-.14, .56], \mathrm{B}_{\mathrm{H}(0,1)}=1.28, \mathrm{RR}_{1 / 3<B<3}[0,5.30]$.

In the synchronous-first group, there was sensitive evidence that synchronous condition drift was greater than zero, $t(63)=1.91, p=0.061,95 \%$ CI $[-0.0,1.4], d=.24$, $95 \%$ CI $[0.03,0.45], \mathrm{B}_{\mathrm{H}(0,1)}=3.10, \mathrm{RR}_{\mathrm{B}>3}[0.33,1.06]$. There was also sensitive evidence that synchronous condition drift was greater than zero in the asynchronous-first group, $t(59)=$ $3.83, p<.001,95 \%$ CI $[0.6,1.9], d=.50,95 \%$ CI $[.27, .72], \mathrm{B}_{\mathrm{H}(0,1)}=445.46 \mathrm{RR}_{\mathrm{B}>3}[.10,>3]$. 


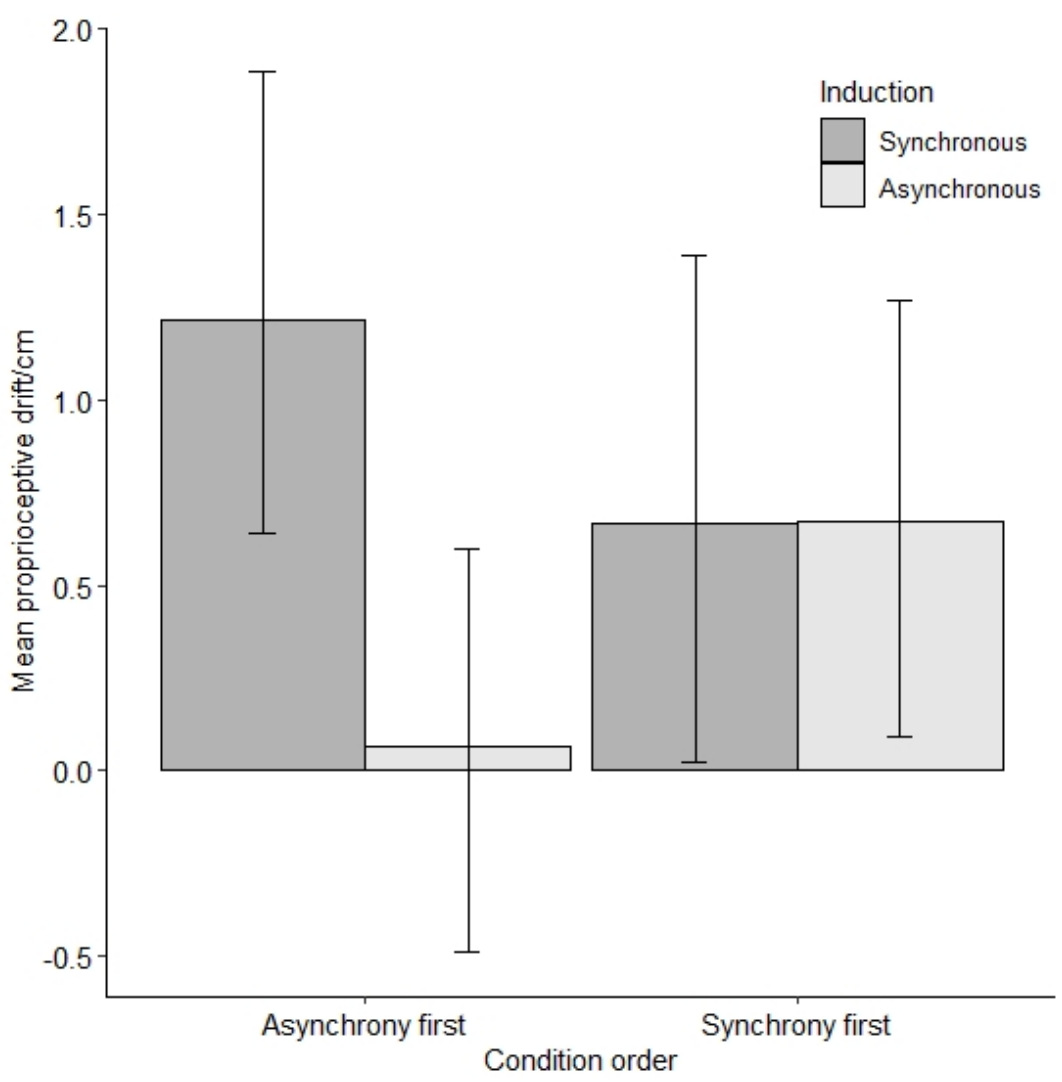

Figure 1. Mean synchronous and asynchronous condition proprioceptive drift in asynchrony-first and synchrony-first groups. Error bars show 95\% CIs.

\section{Subjective report}

\section{Illusion index}

Figure 2 shows mean illusion index (mean of S1 to S3 scores). The difference between synchronous and asynchronous condition illusion index was greater for the asynchrony-first group $(M=2.0, S D=1.9)$ than for the synchrony-first group $(M=1.3, S D=$ $1.7), t(118.39)=2.12, p=.036,95 \% \mathrm{CI}[.05,1.33], d=.3895 \% \mathrm{CI}[.03, .74], \mathrm{B}_{\mathrm{H}(0,1)}=4.93$, $\mathrm{RR}_{\mathrm{B}>3}[0.22,2.0]$. Mean synchronous condition illusion index agreement for the asynchronyfirst group $(M=1.2, S D=1.4)$ was higher than for the synchrony-first group $(\mathrm{M}=0.60, \mathrm{SD}=$ $2.0), t(118.62)=2.17, p=.032,95 \%$ CI $[.05,1.33], d=.3995 \%$ CI $[.03, .74], \mathrm{B}_{\mathrm{H}(0,1)}=4.83$, $\mathrm{RR}_{\mathrm{B}>3}[0.19,1.89]$. 
In the synchronous-first group, there was sensitive evidence that synchronous condition illusion index was greater than zero, $t(63)=2.69, p<.009,95 \% \mathrm{CI}[0.2,1.1], d=$ $.34,95 \% \mathrm{CI}[.12, .55], \mathrm{B}_{\mathrm{H}(0,1)}=14.89 \mathrm{RR}_{\mathrm{B}>3}[0.10,>3]$. There was also sensitive evidence that synchronous condition illusion index was greater than zero in the asynchronous-first group, $t(59)=6.74, p<.001,95 \% \mathrm{CI}[0.9,1.6], d=.87,95 \% \mathrm{CI}[0.6,1.1], \mathrm{B}_{\mathrm{H}(0,1)}=\mathrm{RR}_{\mathrm{B}>3}[0.03,>3]$.

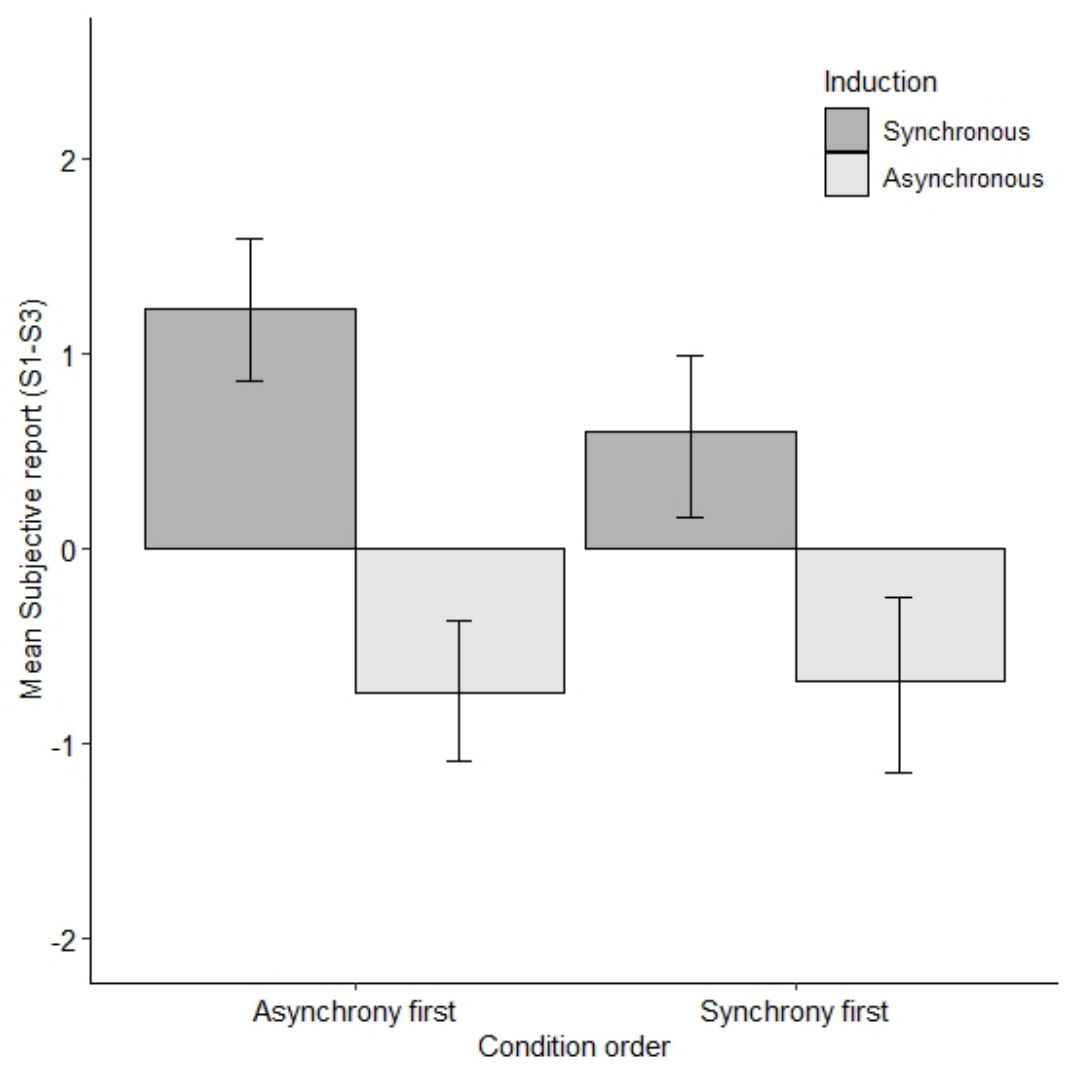

Figure 2. Mean illusion index (combined S1-S3) scores in asynchrony-first and synchronyfirst groups. Error bars show 95\% CIs.

S1: "It seemed as if I were feeling the touch of the paintbrush in the location where I saw the rubber hand touched"

Figure 3 shows mean S1 agreement scores. The difference between synchronous and asynchronous condition S1 (referred touch) agreement was greater for the asynchrony-first group $(M=1.9, S D=2.0)$ than for the synchrony-first group $(\mathrm{M}=1.4, \mathrm{SD}=1.9), t(120.55)$ 
$=1.34, p=0.18,95 \%$ CI $[-0.2,1.2], d=.2495 \% \mathrm{CI}[-.11, .59], \mathrm{B}_{\mathrm{H}(0,1)}=1.33, \mathrm{RR}_{\mathrm{B}>3}[0$,

5.30]. There was also sensitive evidence for a difference in synchronous condition S1

agreement between the asynchrony-first group $(M=2.0, S D=1.2)$ and the synchrony-first

group $(M=1.3, S D=2.0)), t(106.31)=2.26, p=0.026,95 \% \mathrm{CI}[0.1,1.3], d=.4095 \% \mathrm{CI}$

$[.05, .76], \mathrm{B}_{\mathrm{H}(0,1)}=, \mathrm{RR}_{\mathrm{B}>3}=5.58 \mathrm{RR}_{>3}[0.19,2.27]$

Synchronous condition S1 agreement was greater than zero for both the synchronousfirst group, $t(63)=5.25, p<.001,95 \% \mathrm{CI}[0.8,1.8], d=.6695 \% \mathrm{CI}[.43, .88], \mathrm{B}_{\mathrm{H}(0,1)}=$ 163283.44 $\mathrm{RR}_{\mathrm{B}>3}[.06,>3]$, and the asynchronous-first group, $t(59)=12.37, p<.001,95 \% \mathrm{CI}$

$[1.7,2.3], d=1.60,95 \%$ CI $[1.27,1.91], \mathrm{B}_{\mathrm{H}(0,1)}=3.83 \times 10^{29} \mathrm{RR}_{\mathrm{B}>3}[0.02,>3]$.

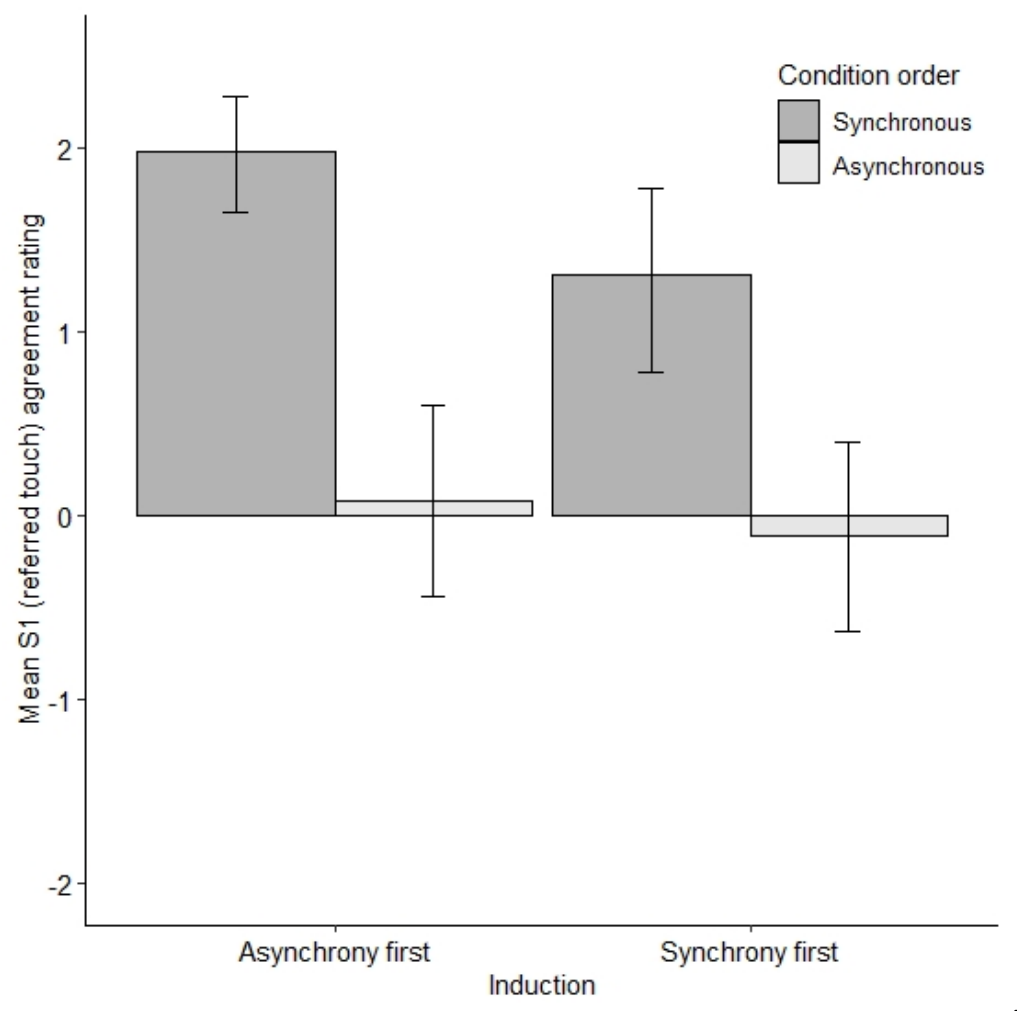

Figure 3. Mean agreement scores for S1 ("It seemed as if I were feeling the touch of the paintbrush in the location where I saw the rubber hand touched") in asynchrony-first and synchrony-first groups. Error bars show 95\% CIs. 
S2: "It seemed as though the touch I felt was caused by the paintbrush touching the rubber hand."

Figure 3 shows mean S2 agreement scores. The difference between synchronous and asynchronous condition S2 (referred touch) agreement was greater for the asynchrony-first group $(\mathrm{M}=2.0, \mathrm{SD}=2.3)$ than for the synchrony-first group $(M=1.3, S D=2.0), t(117.74)=$ $2.03, p=.044,95 \%$ CI $[0.0,1.6], d=.37,95 \% \mathrm{CI}[.01, .72], \mathrm{B}_{\mathrm{H}(0,1)}=4.00, \mathrm{RR}_{\mathrm{B}>3}[0.31$, 1.63]. There was no sensitive evidence for or against a difference in synchronous condition S2 agreement between the asynchrony-first group $(M=1.1, S D=1.8)$ and synchrony-first $\operatorname{group}(M=0.5, S D=2.1), t(120.76)=1.80, p=.075,95 \% \mathrm{CI}[-0.1,1.3], d=.32,95 \% \mathrm{CI}$ $[-.03, .74], \mathrm{B}_{\mathrm{H}(0,1)}=2.74, \mathrm{RR}_{1 / 3<B<3}[0,>6]$.

There was no evidence either way for whether or not synchronous condition S2 agreement was greater than zero in the synchrony first group, $t(63)=1.99, p=.051,95 \% \mathrm{CI}$ $[0.0,1.0], d=.2595 \%$ CI $[.04, .46], \mathrm{B}_{\mathrm{H}(0,1)}=.2 .76 \mathrm{RR}_{\mathrm{B} 1 / 3<\mathrm{B}<3}[.88,>3]$. Synchronous condition S2 agreement score was greater than zero in the asynchrony-first group, $t(59)=$ $5.01, p<.001,95 \%$ CI $[0.7,1.6], d=.6595 \%$ CI $[.41, .88], \mathrm{B}_{\mathrm{H}(0,1)}=23429.74 \mathrm{RR}_{\mathrm{B}>3}[.06,>3]$. 


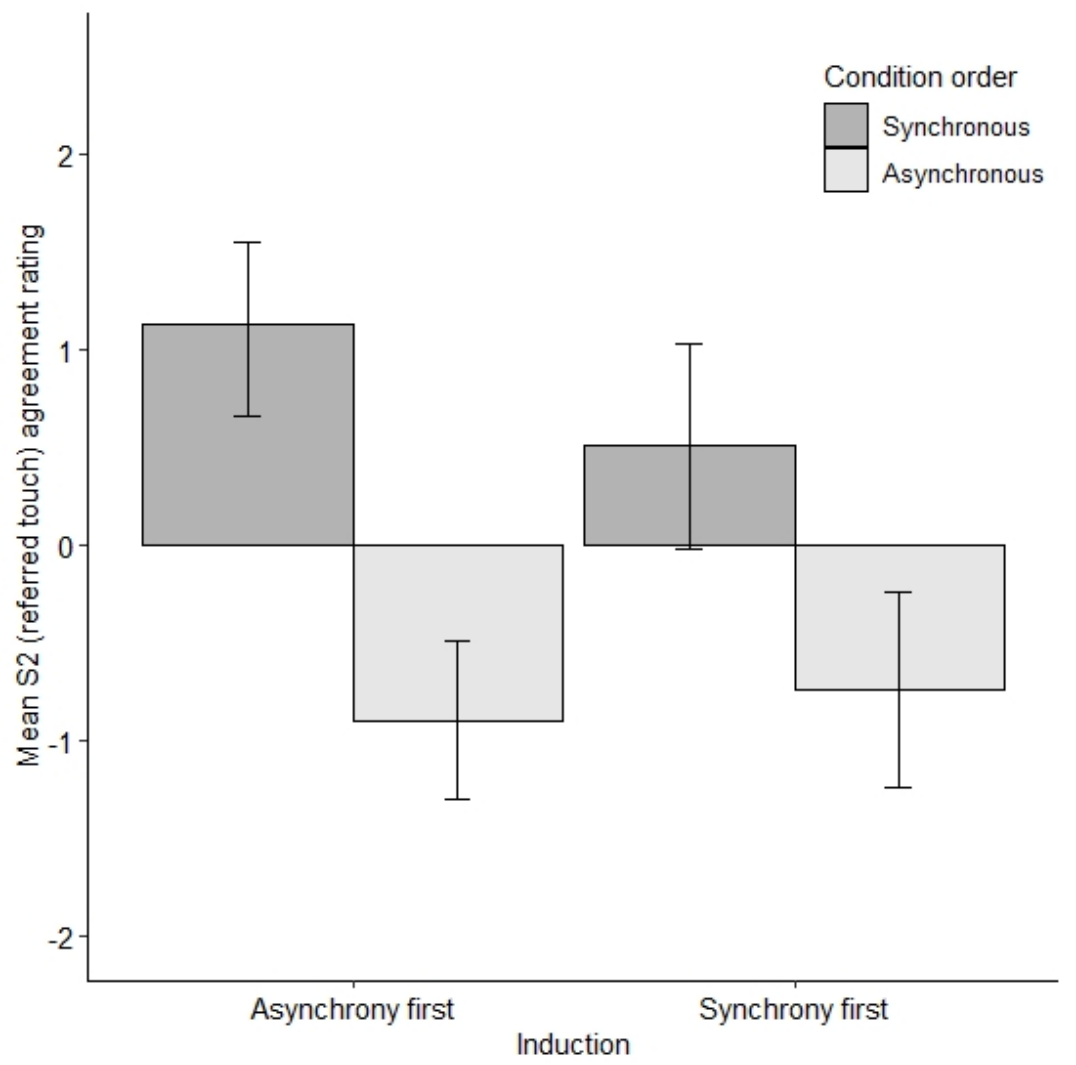

Figure 3. Mean agreement scores for S2 ("It seemed as though the touch I felt was caused by the paintbrush touching the rubber hand.") in asynchrony-first and synchrony-first groups. Error bars show $95 \%$ CIs.

\section{S3: "I felt as if the rubber hand were my hand."}

Figure 4 shows S3 agreement scores. The difference between synchronous and asynchronous condition S3 (ownership) agreement was greater for the asynchrony-first group $(M=2.0, S D=2.2)$ than for the synchrony-first group $(M=1.2, S D=2.0), t(118.06)=2.13, p$ $=.035,95 \% \mathrm{CI}[.06,1.53], d=.3895 \% \mathrm{CI}[.03, .74], \mathrm{B}_{\mathrm{H}(0,1)}=5.32, \mathrm{RR}_{\mathrm{B}>3}[0.25,2.35]$.

There was no sensitive evidence for or against a difference in synchronous condition ownership (S3) agreement between the asynchrony-first group $(M=0.6, S D=1.8)$ and the synchrony-first group $(M=0.0, S D=2.0), t(121.9)=1.70, p=.091,95 \%$ CI $[-0.10,1.30], d$ $=.3195 \% \mathrm{CI}[-.05, .66], \mathrm{B}_{\mathrm{H}(0,1)}=2.32, \mathrm{RR}_{\mathrm{B}>1 / 3<3}[0,>6]$. 
There was sensitive evidence that synchronous condition S3 (ownership) agreement was not greater than zero in the synchrony-first group, $t(63)=-0.06, p=.95,95 \%$ CI $[-.51$, $.48], d=-.0195 \% \mathrm{CI}[-.21, .20], \mathrm{B}_{\mathrm{H}(0,1)}=0.24 \mathrm{RR}_{\mathrm{B}<1 / 3}[.78,>3]$. Conversely, in the asynchrony first group, there was sensitive evidence that synchronous condition S3 agreement was greater than zero, $t(59)=2.35, p=.022,95 \% \mathrm{CI} .1,1.1], d=.3095 \% \mathrm{CI}$ $[.09, .52], \mathrm{B}_{\mathrm{H}(0,1)}=11.31 \mathrm{RR}_{\mathrm{B}<1 / 3}[.12,>3]$.

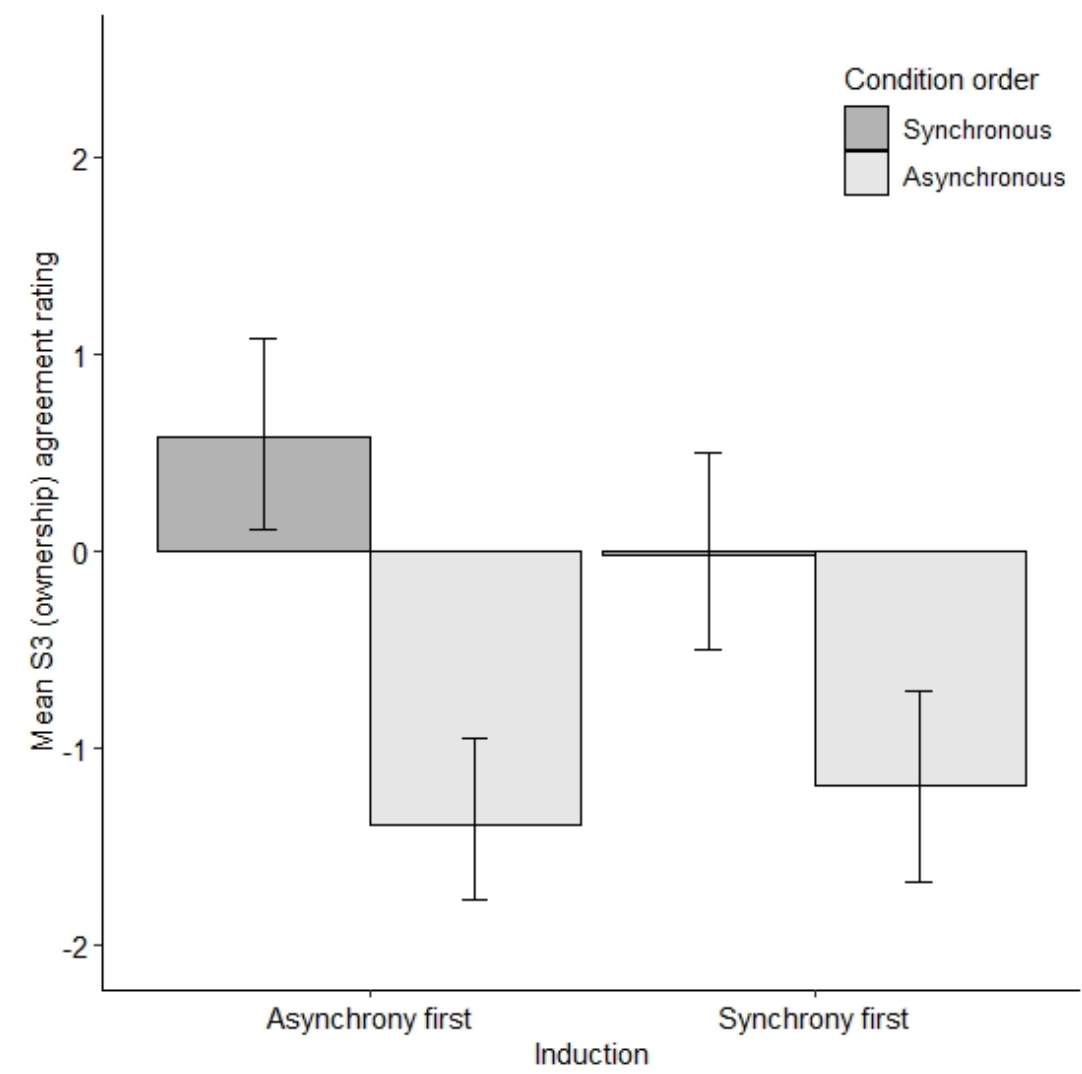

Figure 4. Mean agreement scores for S3 ("I felt as if the rubber hand were my hand.") in asynchrony-first and synchrony-first groups. Error bars show 95\% CIs. 


\section{Frequency of agreement.}

Table 1 shows frequencies of agreement (a Likert scale score equal to or greater than one) for illusion index and each individual statement, split by task order.

Table 1

Percentage of participants reporting agreement for synchronous and asynchronous conditions (split by condition order).

\begin{tabular}{|c|c|c|c|}
\hline & & \multicolumn{2}{|c|}{ Condition order } \\
\hline & & Synchronous-first & Asynchronous-first \\
\hline \multicolumn{4}{|c|}{ Illusion index (S1-S3) } \\
\hline & Synchronous & $54.7 \%$ & $66.7 \%$ \\
\hline & Asynchronous & $26.6 \%$ & $16.7 \%$ \\
\hline \multicolumn{4}{|c|}{ S1 } \\
\hline & Synchronous & $78.1 \%$ & $91.7 \%$ \\
\hline & Asynchronous & $48.4 \%$ & $51.7 \%$ \\
\hline \multicolumn{4}{|c|}{ S2 } \\
\hline & Synchronous & $59.4 \%$ & $73.3 \%$ \\
\hline & Asynchronous & $32.8 \%$ & $28.3 \%$ \\
\hline \multicolumn{4}{|c|}{ 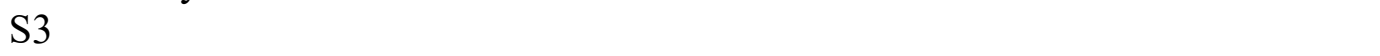 } \\
\hline & Synchronous & $50.0 \%$ & $63.3 \%$ \\
\hline & Asynchronous & $23.4 \%$ & $21.7 \%$ \\
\hline
\end{tabular}

Note. S1: It seemed as if I were feeling the touch of the paintbrush in the location where I saw the rubber hand touched. S2: It seemed as though the touch I felt was caused by the paintbrush touching the rubber hand. S3: I felt as if the rubber hand were my hand. 


\section{Discussion}

Participants who were exposed to the control (asynchronous) condition prior to the experimental (synchronous) condition reported higher illusion index in the synchronous condition, and a greater difference between synchronous and asynchronous condition for both illusion index and proprioceptive drift. A simple explanation for these order effects is that demand characteristics communicated during exposure to the control condition drive hypothesis awareness for the second (experimental) condition, which can be met by a range of responses (e.g., faking, bias or phenomenological control; see Corneille \& Lush, 2021). These results therefore further highlight the role of expectancies in RHI report.

It has been recently argued that, although expectancies are not matched across experimental and control conditions, this does not present a problem for interpretation of effects as reflecting multisensory integration processes, because the difference in report between conditions does not correlate with phenomenological control (Ehrsson et al., 2021). We disagree with this claim because the difference in expectancies between conditions also does not correlate with phenomenological control, and the invariant expectancies can be met by a range of hypothesis awareness effects (e.g., bias; see Lush, Dienes, et al., 2021). The effect of task order on the difference between synchronous and asynchronous measures presented here further attests to the confounding effect of demand characteristics for comparison of synchronous and asynchronous measures in the RHI. Note that while there was evidence for synchronous condition measures beyond order effects, this does not indicate that these are not attributable to demand characteristics (demand characteristics are not restricted to order effects).

The RHI consists of two distinct experiences: ownership of the fake hand and touch referred to the fake hand. One statement (S3) is considered to measure ownership experience 
and two referred touch experience (S1 and S2). When measured in large samples, synchronous condition ownership agreement is weak (e.g., 0.4; Longo et al., 2008)) compared to referred touch agreement (1.9 for S1 and 1.1 for S2; Longo et al., 2008)). Combining these scores into an illusion index therefore not only conflates reports of the two varieties of experience but also inflates ownership scores (Kalckert et al., 2019; Reader et al., 2021; Roseboom \& Lush, 2020). This has important implications when order effects are considered. On average, participants reported ownership experience following synchronous stimulation only if they had previously been exposed to the ownership statement and control condition procedure. Note also that, while the RHI is typically reported to occur in $70 \%$ of participants (Riemer et al., 2019), just 50\% of participants who were not previously exposed to the ownership statement reported ownership experience (compare to the approximately $50 \%$ of this group who reported referred touch experience in the asynchronous control condition). Given the substantial relationship between phenomenological control (trait response to imaginative suggestion) and ownership agreement scores (Lush, Dienes, et al., 2021), these data are consistent with experience of ownership of a fake hand being at least partly attributable to phenomenological control (along with other hypothesis awareness effects; see Corneille \& Lush, 2021).

What might explain the relatively high agreement generated by the referred touch statement S1 compared to S2 (which also describes referred touch) in large sample studies (see Longo et al., 2008; Lush et al., 2020)? It has previously been noted (Wu, in press) that $\mathrm{S} 1$ is semantically ambiguous ("the location" could refer to touch felt on the fake or real hand) and is sometimes interpreted as a check that the brush strokes are concurrent (i.e., that the experimenter is doing a decent job of applying the stimuli; see Roseboom \& Lush, 2020). Therefore, while S1 agreement was greater than zero in the synchronous-first group, it is unsafe to interpret this as evidence of an experience of referred touch beyond order effects. 
For the unambiguously phrased referred touch statement (S2), the data were insensitive as to whether or not there was evidence for agreement scores greater than zero in the absence of order effects.

In conclusion, analysis of existing open data shows order effects in RHI measures. This supports the role of demand characteristics in the rubber hand illusion (including phenomenological control effects, Lush et al., 2020). The analyses reported here were not preregistered and should therefore be considered exploratory. Furthermore, effects seen in this non-naïve sample may differ from effects found in participants with no prior knowledge of the RHI. Although testing these possibilities will require further research, fortunately there is a large body of existing RHI data for which order effects could be compared with the results presented here. Systematically examining order effects in this larger data set would advance our understanding of the contribution of demand characteristics to RHI effects. 


\section{References}

Alsmith, A. (2015). Mental Activity \& the Sense of Ownership. Review of Philosophy and Psychology, 6(4), 881-896. https://doi.org/10.1007/s13164-014-0208-1

Botan, V., Fan, S., Critchley, H., \& Ward, J. (2018). Atypical susceptibility to the rubber hand illusion linked to sensory-localised vicarious pain perception. Consciousness and Cognition, 60, 62-71. https://doi.org/10.1016/j.concog.2018.02.010

Botvinick, M., \& Cohen, J. (1998). Rubber hands 'feel' touch that eyes see. Nature, 391(6669), 756-756. https://doi.org/10.1038/35784

Chancel, M., Ehrsson, H. H., \& Ma, W. J. (2021). Uncertainty-based inference of a common cause for body ownership. OSF Preprints. https://doi.org/10.31219/osf.io/yh2z7

Corneille, O., \& Lush, P. (2021). Sixty years after Orne's American Psychologist article: A conceptual analysis of "Demand Characteristics". PsyArXiv. https://doi.org/10.31234/osf.io/jqyvx

Dieguez, S. (2018). The illusion illusion. Constructivist Foundations, 14, 108-110.

Dienes, Z. (2019). How Do I Know What My Theory Predicts? Advances in Methods and Practices in Psychological Science, 2(4), 364-377. https://doi.org/10.1177/2515245919876960

Dienes, Z., Lush, P., Palfi, B., Roseboom, W., Scott, R., Parris, B., Seth, A., \& Lovell, M. (2020). Phenomenological control as cold control. Psychology of Consciousness: Theory, Research, and Practice, No Pagination Specified-No Pagination Specified. https://doi.org/10.1037/cns0000230 
Durgin, F. H., Evans, L., Dunphy, N., Klostermann, S., \& Simmons, K. (2007). Rubber Hands Feel the Touch of Light. Psychological Science, 18(2), 152-157. https://doi.org/10.1111/j.1467-9280.2007.01865.x

Ehrsson, H. H., Fotopoulou, A., Radziun, D., Longo, M., \& Tsakiris, M. (2021). No specific relationship between hypnotic suggestibility and the rubber hand illusion: A commentary with new and verification analyses. PsyArXiv. https://doi.org/10.31234/osf.io/pmzgc

Kalckert, A., Bico, I., \& Fong, J. X. (2019). Illusions With Hands, but Not With Balloons Comparing Ownership and Referral of Touch for a Corporal and Noncorporal Object After Visuotactile Stimulation. Perception, 48(5), 447-455. https://doi.org/10.1177/0301006619839286

Klein, O., Doyen, S., Leys, C., Magalhães de Saldanha da Gama, P. A., Miller, S., Questienne, L., \& Cleeremans, A. (2012). Low Hopes, High Expectations: Expectancy Effects and the Replicability of Behavioral Experiments. Perspectives on Psychological Science, 7(6), 572-584. https://doi.org/10.1177/1745691612463704

Longo, M. R., Schüür, F., Kammers, M. P. M., Tsakiris, M., \& Haggard, P. (2008). What is embodiment? A psychometric approach. Cognition, 107(3), 978-998. https://doi.org/10.1016/j.cognition.2007.12.004

Lush, P. (2020). Demand Characteristics Confound the Rubber Hand Illusion. Collabra: Psychology, 6(1), 22. https://doi.org/10.1525/collabra.325

Lush, P., Botan, V., Scott, R. B., Seth, A. K., Ward, J., \& Dienes, Z. (2020). Trait phenomenological control predicts experience of mirror synaesthesia and the rubber hand illusion. Nature Communications, 11(1), 4853. https://doi.org/10.1038/s41467020-18591-6 
Lush, P., Dienes, Z., \& Seth, A. (2021). Rubber hand illusion report is confounded by demand characteristics and may entirely reflect compliance, bias and implicit imaginative suggestion effects. PsyArXiv. https://doi.org/10.31234/osf.io/qh4ag

Lush, P., Seth, A., \& Dienes, Z. (2021). Demand characteristics confound asynchronous control conditions in indirect measures of the rubber hand illusion. PsyArXiv. https://doi.org/10.31234/osf.io/w67xc

Orne, M. T. (1962). On the social psychology of the psychological experiment: With particular reference to demand characteristics and their implications. American Psychologist, 17(11), 776-783. http://dx.doi.org/10.1037/h0043424

Reader, A. T. (2021). What do participants expect to experience in the rubber hand illusion? PsyArXiv. https://doi.org/10.31234/osf.io/d8x9y

Reader, A. T., Trifonova, V. S., \& Ehrsson, H. H. (2021). The Relationship Between Referral of Touch and the Feeling of Ownership in the Rubber Hand Illusion. Frontiers in Psychology, 12, 629590. https://doi.org/10.3389/fpsyg.2021.629590

Riemer, M., Trojan, J., Beauchamp, M., \& Fuchs, X. (2019). The rubber hand universe: On the impact of methodological differences in the rubber hand illusion. Neuroscience \& Biobehavioral Reviews, 104, 268-280. https://doi.org/10.1016/j.neubiorev.2019.07.008

Roseboom, W., \& Lush, P. (2020). Serious problems with interpreting rubber hand illusion experiments [Preprint]. PsyArXiv. https://doi.org/10.31234/osf.io/uhdzs

Sharpe, D., \& Whelton, W. J. (2016). Frightened by an Old Scarecrow: The Remarkable Resilience of Demand Characteristics. Review of General Psychology, 20(4), 349368. https://doi.org/10.1037/gpr0000087 
ORDER EFFECTS IN THE RUBBER HAND ILLUSION

Wu, W. (in press). Mineness and introspective data. In M. Guillot \& M. G.- Carpintero (Eds.), The Sense of Mineness. Oxford University Press. 
ORDER EFFECTS IN THE RUBBER HAND ILLUSION

\section{Acknowledgements}

Thanks to Zoltan Dienes, Anil Seth and Olivier Corneille for helpful feedback on this manuscript. 